International Journal of Pure and Applied Mathematics

Volume 104 No. 3 2015, 353-362

ISSN: 1311-8080 (printed version); ISSN: 1314-3395 (on-line version)

url: http://www.ijpam.eu

doi: http://dx.doi.org/10.12732/ijpam.v104i3.6

ijpam.eu

\title{
OPTIMAL CONSUMPTION AND PORTFOLIO DECISIONS WHEN THE RISKY ASSET IS DRIVEN BY \\ A TIME-INHOMOGENEOUS MARKOV MODULATED DIFFUSION PROCESS
}

\author{
Benjamín Vallejo-Jiménez ${ }^{1}$, Francisco Venegas-Martínez ${ }^{2}$, \\ Yazmín Viridiana Soriano-Morales ${ }^{3}$ \\ ${ }^{1,2,3}$ Escuela Superior de Economía of the Instituto Politécnico Nacional \\ Plan de Agua Prieta No. 66, Miguel Hidalgo \\ C.P. 11340 \\ Mexico City, MÉXICO
}

\begin{abstract}
This research is aimed at finding closed-form solutions of the utility maximization problem that solves an infinitely-lived rational consumer when the risky asset is driven by a time-inhomogeneous Markov modulated diffusion process. We provide analytical solutions for two specific cases: transition probabilities with tendency and transition probabilities with no tendency and no periodicity.
\end{abstract}

AMS Subject Classification: 35F21, 60J27, 60G50, 60J60

Key Words: diffusion processes, HamiltonJacobiBellman equation, continuoustime Markov processes

\section{Introduction}

The problem of an infinitely-lived rational consumer maximizing his/her total discounted utility when a portfolio of risky assets is considered has been widely studied since the pioneer paper of Merton [6], see for instance Stockbridge [9]

Received: June 8, 2015

(C) 2015 Academic Publications, Ltd.

$\S$ Correspondence author url: www.acadpubl.eu 
and Venegas-Martínez [10]. In his work Merton used diffusion process to model the dynamics of asset returns, and many extensions have developed since then. Currently, much of recent work in this subject aims at modeling the stock price by a Markov Modulated process, see for instance: Fei [3] studying optimal consumption and portfolio allocation under ination with a Markov-switching model; Sotomayor and Cadenillas [8] providing explicit solutions for the optimal consumption-investment decisions according to a specific Hyperbolic Absolute Risk Aversion (HARA) utility function when asset prices are driven by a standard Brownian motion combined with a regime switching; and finally, Buerle and Rieder [1] obtaining the optimal consumption and portfolio allocations when the stock return and its volatility depend on an external homogeneous and finite Markov chain. In particular, Buerle and Rieder [1] focus on the problem of maximizing the total expected utility from terminal wealth and solve it by stochastic control methods for different functional forms of utility functions. It is also worth mentioning Zariphopoulous [11] work on maximizing expected utility from consumption and terminal wealth under the assumption that the risky asset depends on a continuous-time Markov chain.

At the best of our knowledge, regime switching models were originally proposed by Hamilton [4] to model stock return time series. Subsequently, Di Masi et al. [2] provide the first attempt to model volatility as a continuous-time Markov chain, leading to a better representation of the financial reality than the traditional models with deterministic parameters; however, this approach brought new difficulties, especially in the model proposed by Hamilton [4], due to the additional source of uncertainty affecting the completeness of the market.

The approach to find the consumption and portfolio optimal decisions with incomplete regime switching models considering partial information has also been broadly studied, for instance: Stockbridge [9] bringing a linear programming formulation of the portfolio optimization problem with regime switching; Zhang and Yin [12] offering nearly optimal strategies in a financial market with regime switching; and, finally, Sass and Haussmann [7] solving numerically the problem of maximizing the investors expected utility of terminal wealth under a finite time horizon. It is worth mentioning that Di Masi et al. [2] approach has the advantage that many portfolio problems can be solved explicitly and a diffusion process can be approached arbitrarily closely by a continuous-time Markov chain (see also, Kushner and Dupuis [5]).

This investigation extends Buerle and Rieders [1] research in finding analytical solutions for the case of total discounted logarithmic utility when the risky asset is driven by a drift modulated time-inhomogeneous finite Markov chain. The main contribution of this work consists of providing closed-form solution when 
the drift and the volatility of the risky asset are modulated by a time-dependent Markov chain. Additionally, we carry out the application of two different tendency cases: the first one with a transition probability with tendency, and the second one with a transition probability with no tendency and no periodicity. This research is organized as follows: in section 2, we setup the mathematical framework of the proposed model; in section 3, we provide the analytical solution of optimal consumption and asset allocation: through section 4, we study a specific case with transition probabilities with tendency: in section 5, we obtain analytic solution with transition probabilities with no tendency and no periodicity; and, finally, in section 6 we present the conclusion and acknowledge limitations.

\section{The Setup of the Model}

We first state the problem of determining consumption and portfolio decisions that maximize the total discounted expected utility of an infinitely-lived rational consumer when the risky asset is driven by a time-inhomogeneous Markov chain. The consumer has access to a bond and a risky asset. The randomness in the risky asset involves a filtered probability space (or stochastic basis)

$$
\left(\Omega, \mathcal{F}, \mathfrak{F}=\left\{\mathcal{F}_{t}, 0 \leq t \leq T\right\}, P\right)
$$

i.e., $\Omega$ is a sample space $(\mathbb{R}), \mathcal{F}$ is a $\sigma$-algebra on $\Omega$ (the Borel $\sigma$-algebra $B(\mathbb{R})$ ), $P$ is a probability measure on $(\Omega, \mathcal{F})$ and $\mathcal{F}_{t}$ is a filtration containing all information of the market until time $t$. The bond price process $b_{t}$ evolves according to

$$
\frac{\mathrm{d} b_{t}}{b_{t}}=r \mathrm{~d} t
$$

and the stock price process $S_{t}$ is driven by a Markov modulated diffusion process

$$
\frac{\mathrm{d} S_{t}}{S_{t}}=\mu_{i} \mathrm{~d} t+\sigma_{i} \mathrm{~d} W_{t}
$$

where $\mathrm{d} W_{t} \sim N(0, \mathrm{~d} t)$ is a standard Brownian motion, $\left(\mu_{i}, \sigma_{i}\right)$ is a continuoustime Markov chain with finite state space $E$, and $Q=\left(q_{i j}(t)\right)_{i, j \in E}$ is a matrix providing the time dependent transition probability under $P$ with respect to $\mathfrak{F}$. In what follows, we suppose that $\mu_{i}, \sigma_{i}: E \rightarrow \mathbb{R}$ and $\sigma_{i}>0$ for all $i \in E$, allowing random changes of the return rate $\mu_{i}$ and in the volatility $\sigma_{i}$.

From now on, we will denote by $\theta_{t}$ the proportion of wealth, in real terms, destined for stock at time $t$. The process $\theta_{t}$ is called portfolio strategy. A 
portfolio strategy is called admissible whenever $\int_{0}^{T} \theta_{s}{ }^{2} \mathrm{ds}<\infty$ almost surely. Let us denote by $a_{t}$ the real wealth process under the self-financing assumption, therefore

$$
\mathrm{d} a_{t}=a_{t}\left(1-\theta_{t}\right) \frac{\mathrm{d} b_{t}}{b_{t}}+a_{t} \theta_{t} \frac{\mathrm{d} S_{t}}{S_{t}}-c_{t} \mathrm{~d} t
$$

with $a_{0}>0$. Consider a consumer-investor that has a strictly concave and increasing utility function $u:[0, \infty) \rightarrow \mathbb{R}$. The individual has a subjective discount rate, $\rho$, and wishes to maximize his/her total expected discount utility given by

$$
\mathrm{E}\left[\int_{0}^{\infty} u\left(c_{t}\right) e^{-\rho t} \mathrm{~d} t \mid F_{t}\right]
$$

subject to the following consolidated budget constraint

$$
\mathrm{d} a_{t}=a_{t}\left(r+\theta_{t}\left(\mu_{i}-r\right)-\frac{c_{t}}{a_{t}}\right) \mathrm{d} t+a_{t} \theta_{t} \sigma_{i} d W_{t} .
$$

In order to use the Hamilton-Jacobi-Bellman (HJB) approach, we define, as usual, the value function

$$
J\left(a_{t}, t, i\right)=\max _{\left.c_{s}\right|_{s \in[t, \infty)}} \mathrm{E}\left[\int_{t}^{\infty} u\left(c_{s}\right) e^{-\rho s} \mathrm{~d} s\right]
$$

Then,

$$
\begin{aligned}
& J\left(a_{t}, t, i\right)=\max _{\left.c_{s}\right|_{s \in[t, t+\mathrm{d} t]}} \mathrm{E}\left\{\int_{t}^{t+\mathrm{d} t} u\left(c_{s}\right) e^{-\rho s} \mathrm{~d} s+\left.\max _{c_{z} \mid}\right|_{z \in[t+d t, \infty)} \mathrm{E}\left[\int_{t+\mathrm{d} t}^{\infty} u\left(c_{z}\right) e^{-\rho z} \mathrm{~d} z\right]\right\} \\
& \max _{\left.c_{s}\right|_{s \in[t, t+\mathrm{d} t]}} \mathrm{E}\left[\int_{t}^{t+\mathrm{d} t} u\left(c_{s}\right) e^{-\rho s} \mathrm{~d} s+J\left(a_{t}+\mathrm{d} a_{t}, t+d t, i\right)\right] \\
& \max _{\left.c_{s}\right|_{s \in[t, t+\mathrm{d} t]}} \mathrm{E}\left[u\left(c_{t}\right) e^{-\rho t} \mathrm{~d} t+\mathrm{d} J\left(a_{t}, t, i\right)+J\left(a_{t}, t, i\right)+\mathrm{o}(d t)\right]
\end{aligned}
$$

On the other hand, if we rewrite the budget constraint as

$$
\mathrm{d} a_{t}=a_{t} \mu_{a} \mathrm{~d} t+a_{t} \sigma_{a} \mathrm{~d} W_{t}
$$


where $\mu_{a}=r+\theta_{t}\left(\mu_{i}-r\right)-\frac{c_{t}}{a_{t}}$ and $\sigma_{a}=\theta_{t} \sigma_{i}$. Then, Itôs lemma for Markov chain modulated diffusion process with (1) for the underlying process leads to

$$
\begin{aligned}
0= & \max _{\left.c_{s}\right|_{s \in[t, t+\mathrm{d} t]}} \mathrm{E}\left\{u\left(c_{t}\right) e^{-\rho t} \mathrm{~d} t+o(d t)+\frac{\partial J\left(a_{t}, t, i\right)}{\partial a_{t}} a_{t} \sigma_{a} \mathrm{~d} W_{t}\right. \\
& +\left(\frac{\partial J\left(a_{t}, t, i\right)}{\partial t}+\frac{\partial J\left(a_{t}, t, i\right)}{\partial a_{t}} a_{t} \mu_{a}+\frac{1}{2} \frac{\partial^{2} J\left(a_{t}, t, i\right)}{\partial a_{t}{ }^{2}} a_{t}^{2} \sigma_{a}^{2}\right) \mathrm{d} t \\
& +\left(\sum_{j \in E} q_{i j}(t)\left[J\left(a_{t}, t, j\right)-J\left(a_{t}, t, i\right)\right] j \mathrm{~d} t\right\} .
\end{aligned}
$$

If $c_{t}$ and $\theta_{t}$ are both optimal, then

$$
\begin{aligned}
0= & u\left(c_{t}\right) e^{-\rho t}+\frac{\partial J\left(a_{t}, t, i\right)}{\partial t}+\frac{\partial J\left(a_{t}, t, i\right)}{\partial a_{t}} a_{t}\left(r+\theta_{t}\left(\mu_{i}-r\right)-\frac{c_{t}}{a_{t}}\right) \\
& +\frac{1}{2} \frac{\partial^{2} J\left(a_{t}, t . i\right)}{\partial a_{t}{ }^{2}} a_{t}{ }^{2} \theta_{t}^{2} \sigma_{i}^{2}+\sum_{j \in E} q_{i j}(t)\left[J\left(a_{t}, t, j\right)-J\left(a_{t}, t, i\right)\right] .
\end{aligned}
$$

A suitable candidate that meets the previously established condition is given by

$$
S\left(a_{t}, t, i\right)=\left[\beta_{0}+\beta_{1} u\left(a_{t}\right)\right] e^{-\rho t}+g(t, i) e^{-\rho t}
$$

Indeed, by substituting (3) in (2), we have

$$
\begin{aligned}
0= & u\left(c_{t}\right)-\rho\left(\beta_{0}+\beta_{1} u\left(a_{t}\right)\right)+\frac{\partial g(t, i)}{\partial t}-\rho g(t, i) \\
& +\beta_{1} u^{\prime}\left(a_{t}\right) a_{t}\left(r+\theta_{t}\left(\mu_{i}-r\right)-\frac{c_{t}}{a_{t}}\right)+\frac{1}{2} \beta_{1} u^{\prime \prime}\left(a_{t}\right) a_{t}^{2} \theta_{t}^{2} \sigma_{i}^{2} \\
& +\sum_{j \in E} q_{i j}(t)[g(t, j)-g(t, i)] .
\end{aligned}
$$

After differentiating the above expression with respect to $c_{t}$ and $\theta_{t}$, respectively, we obtain

$$
u^{\prime}\left(c_{t}\right)=\beta_{1} u^{\prime}\left(a_{t}\right) \text { and } \theta_{t}=\frac{\left[\left(\mu_{i}-r\right) / \sigma_{i}^{2}\right]}{-\left[u^{\prime \prime}\left(a_{t}\right) a_{t} / u^{\prime}\left(a_{t}\right)\right]} .
$$

Where $\left(\mu_{i}-r\right) / \sigma_{i}^{2}$ is the risk premium adjusted by volatility in the state $i$, and $-u^{\prime \prime}\left(a_{t}\right) a_{t} / u^{\prime}\left(a_{t}\right)$ is the relative risk aversion degree (being this the elasticity of the marginal utility of wealth, which is related to the Arrow-Pratt measure). 


\section{Analytic Solution for Logarithmic Utility}

The present section is focused in finding an analytical solution of the optimal asset allocation problem with logarithmic utility, $u(c)=\ln (c)$. First, we characterize optimal decisions. Note that necessary conditions for a maximum lead to

$$
c_{t}=\frac{1}{\beta_{1}} a_{t} \text { and } \theta_{t}=\frac{\mu_{i}-r}{\sigma_{i}^{2}}
$$

We observe that does not depend on $t$, it just depends on the state $i$, thus it is convenient to change notation to $\theta_{i}=\frac{\mu_{i}-r}{\sigma_{i}^{2}}$. By substituting (5) in (4), we have

$$
\begin{aligned}
0= & r \beta_{1}-\ln \left(\beta_{1}\right)-1-\rho \beta_{0}+\left(1-\rho \beta_{1}\right) \ln \left(a_{t}\right) \\
& +\frac{\partial g(t, i)}{\partial t}-\rho \mathrm{g}(t, i)+\frac{1}{2} \beta_{1} \lambda_{i}^{2}+\sum_{j \in E} q_{i j}(t)[g(t, j)-g(t, i)]
\end{aligned}
$$

We note that this equation must hold for any value of $a_{t}$, and since the rest of the equation does not depend on that variable, then $1-\rho \beta_{1}=0$ or $\beta_{1}=\rho^{-1}$, thus,

$$
c_{t}=\rho a_{t} .
$$

This gives the exact marginal propensity to consumption at all time. Therefore,

$$
\begin{aligned}
0= & \frac{r}{\rho}+\ln (\rho)-1-\rho \beta_{0}+\frac{\partial g(t, i)}{\partial t}-\rho g(t, i) \\
& +\frac{1}{2 \rho} \lambda_{i}^{2}+\sum_{j \in E} q_{i j}(t)[g(t, j)-g(t, i)] .
\end{aligned}
$$

Now it is clear that there is a part of the equation that does not depend on the state, then the equation can be split in two parts which are equal to zero. That is,

$$
0=\frac{r}{\rho}+\ln (\rho)-1-\rho \beta_{0}
$$

and

$$
0=\frac{\partial g(t, i)}{\partial t}-\rho g(t, i)+\frac{1}{2 \rho} \lambda_{i}^{2}+\sum_{j \in E} q_{i j}(t)[g(t, j)-g(t, i)]
$$

After solving (6) for $\beta_{0}$, we get

$$
\beta_{0}=\rho^{-1}\left(\frac{r}{\rho}+\ln (\rho)-1\right)
$$


In order to solve (7), we propose as a candidate

$$
\begin{aligned}
g(t, i) & =\int_{t}^{\infty} \frac{1}{2 \rho} \lambda_{i}^{2} e^{-\rho(s-t)} \mathrm{d} s+\sum_{j \in E} \int_{t}^{\infty} q_{i j}(s)[g(s, j)-g(s, i)] e^{-\rho(s-t)} \mathrm{d} s \\
& =\frac{1}{2 \rho^{2}} \lambda_{i}^{2} e^{\rho t} \int_{t}^{\infty} \rho e^{-\rho s} \mathrm{~d} s+e^{\rho t} \sum_{j \in E} \int_{t}^{\infty} q_{i j}(s)[g(s, j)-g(s, i)] e^{-\rho s} \mathrm{~d} s
\end{aligned}
$$

where $q_{i j}(t)$ and $g(t, i)$ are integrable on $t$ for every interval contained in $[0, \infty)$. The partial derivative of (8) with respect to $t$ leads to

$$
\begin{aligned}
\frac{\partial g(t, i)}{\partial t}= & \rho\left(\int_{t}^{\infty} \frac{1}{2 \rho} \lambda_{i}^{2} e^{-\rho(s-t)} \mathrm{d} s+\sum_{j \in E} \int_{t}^{\infty} q_{i j}(s)[g(s, j)-g(s, i)] e^{-\rho(s-t)} \mathrm{d} s\right) \\
& -\left(\frac{1}{2 \rho} \lambda_{i}^{2}+\sum_{j \in E} q_{i j}(t)[g(t, j)-g(t, i)]\right) .
\end{aligned}
$$

By substituting $g(t, i)$ in the above expression, we have

$$
\frac{\partial g(t, i)}{\partial t}-\rho g(t, i)+\frac{1}{2 \rho} \lambda_{i}^{2}+\sum_{j \in E} q_{i j}(t)[g(t, j)-g(t, i)]=0
$$

Hence, the proposed function fulfills the conditions to solve analytically the stated decision making problem.

\section{Case A: Transition probability with tendency to stability}

In this section, we study a specific case of a time-dependent Markov chain with transition probabilities with tendency. In particular, consider a two-state set E with transition probabilities defined by

$$
q_{11}(t)=1-e^{-\xi_{1} t}, q_{12}(t)=e^{-\xi_{1} t}, q_{21}(t)=e^{-\xi_{2} t} \text { and } q_{22}(t)=1-e^{-\xi_{2} t}
$$

with $\xi_{i}>0, i=1,2$. In this case, the transition probabilities are stabilized at rate $\xi_{i}$ as time evolves. As $t$ tends to infinity, the probability of maintaining the state where it is tends to 1 . In this case, the proposed $g(t, i)$ is given by

$$
g(t, i)=\int_{t}^{\infty} \frac{1}{2 \rho} \lambda_{i}^{2} e^{-\rho(s-t)} \mathrm{d} s+\int_{t}^{\infty} e^{-\xi_{i} s}[g(s, j)-g(s, i)] e^{-\rho(s-t)} \mathrm{d} s
$$


The partial derivative of (9) with respect to $t$ leads to

$$
\begin{aligned}
\frac{\partial g(t, i)}{\partial t}= & \rho\left(\int_{t}^{\infty} \frac{1}{2 \rho} \lambda_{i}^{2} e^{-\rho(s-t)} \mathrm{d} s+\int_{t}^{\infty} e^{-\xi_{i} s}[g(s, j)-g(s, i)] e^{-\rho(s-t)} \mathrm{d} s\right. \\
& -\left(\frac{1}{2 \rho} \lambda_{i}^{2}+e^{-\xi_{i} t}[g(t, j)-g(t, i)]\right)
\end{aligned}
$$

By substituting $g(t, i)$ in (10), produces

$$
\frac{\partial g(t, i)}{\partial t}-\rho g(t, i)+\frac{1}{2 \rho} \lambda_{i}^{2}+e^{-\xi_{i} t}[g(t, j)-g(t, i)]=0
$$

Hence, the proposed candidate fulfills all the required conditions to solve the analytically the stated utility maximization problem.

\section{Case B: Transition probability with no tendency and no periodicity}

While case A explores a transition probability tending to be stationary with speed $\xi_{i}>0, i=1,2$, case B will examine the possibility of a non-stationary transition probability that do not have defined periods. Consider the logistic mapping

$$
x_{n+1}=4 x_{n}\left(1-x_{n}\right)
$$

which has a closed-form solution

$$
x_{n}=\sin ^{2}\left(2^{n-1} \cos ^{-1}\left(1-2 x_{0}\right)\right)
$$

Equation (11) is a mapping taking values in $[0,1]$, which is useful for modeling probabilities, as well as for providing no cycles (periods) nor tendencies. In particular, consider a two-state set $E$ with transition probabilities defined by $q_{11}(t)=1-\sin ^{2}\left(2^{t} \xi_{1}\right), q_{12}(t)=\sin ^{2}\left(2^{t} \xi_{1}\right), q_{21}(t)=\sin ^{2}\left(2^{t} \xi_{2}\right)$ and $q_{22}(t)=$ $1-\sin ^{2}\left(2^{t} \xi_{2}\right)$

with $\xi_{i} \in(0,1), i=1,2$, where for the sake simplicity we have chosen different initial values of $x_{0}$ for each state $i$, in such a way that $\cos ^{-1}\left(1-2 x_{0}\right)=\xi_{i}$. Thus, these transition probabilities fulfill the required conditions. An additional benefit of this is that we know the analytic form ensures the integrability of $q_{i j}(t)$ in any interval of $[0, \infty)$. In this case, the proposed $\mathrm{g}(t, i)$ is given by

$$
g(t, i)=\frac{1}{2 \rho^{2}} \lambda_{i}^{2} e^{\rho t} \int_{t}^{\infty} \rho e^{-\rho s} \mathrm{~d} s+e^{\rho t} \int_{t}^{\infty} \sin ^{2}\left(2^{s} \xi_{i}\right)[g(s, j)-g(s, i)] e^{-\rho s} \mathrm{~d} s
$$


where $\sin ^{2}\left(2^{t} \xi_{i}\right)$ is integrable on $t$ for every interval in $[0, \infty)$. The partial derivative of (12) with respect to $t$ leads to

$$
\begin{aligned}
\frac{\partial \mathrm{g}(t, i)}{\partial t}= & \rho\left(\int_{t}^{\infty} \frac{1}{2 \rho} \lambda_{i}^{2} e^{-\rho(s-t)} d s+\int_{t}^{\infty} \sin ^{2}\left(2^{s} \xi_{i}\right)[\mathrm{g}(s, j)-\mathrm{g}(s, i)] e^{-\rho(s-t)} d s\right) \\
& -\left(\frac{1}{2 \rho} \lambda_{i}^{2}+\sin ^{2}\left(2^{s} \xi_{i}\right)[\mathrm{g}(t, j)-\mathrm{g}(t, i)]\right) .
\end{aligned}
$$

After substituting $g(t, i)$ in (13), and rearranging terms, we obtain

$$
\frac{\partial g(t, i)}{\partial t}-\rho g(t, i)+\frac{1}{2 \rho} \lambda_{i}^{2}+\sin ^{2}\left(2^{s} \xi_{i}\right)[g(t, j)-g(t, i)]=0
$$

Hence, the proposed function fulfills all required conditions.

\section{Conclusions}

This research has provided analytical solutions for the optimal asset allocation problem defined by an infinitely-lived rational consumer with logarithmic utility assuming that the price of the risk asset is driven by a time-inhomogeneous Markov modulated diffusion process. This work also provided closed-form solutions of the optimal consumption and asset allocation of bonds and risky asset holdings at any time. Additionally, this research examined two particular cases of transition probabilities: with tendency, and with no tendency and no periodicity.

\section{References}

[1] N. Buerle, and U. Rieder, Portfolio optimization with Markov-modulated stock prices and interest rates, IEEE Transactions on Automatic Control, 49 (2004), 442-447.

[2] G. B. Di Masi, Y. M. Kabanov and W. J. Runggaldier, Mean-Variance Hedging Of Options on Stocks with Markov Volatilities, Theory of Probability $\&$ Its Applications, 39 (1994), 211-222.

[3] W. Y. Fei, Optimal consumption and portfolio under ination and Markovian switching, Stochastics An International Journal of Probability and Stochastic Processes, 85 (2013), 272-285. 
[4] J. Hamilton, A New Approach to the Economic Analysis of Nonstationary Time Series and the Business Cycle, Econometrica, 57 (1989), 357-384.

[5] H. J. Kushner and P. G. Dupuis, Numerical methods for stochastic control problems in continuous time, Springer, New York (2001).

[6] R. C. Merton, Optimum consumption and portfolio rules in a continuoustime model, Journal of Economic Theory, 3 (1971), 373-413.

[7] J. Sass, and U. G. Haussmann, Optimizing the Terminal Wealth under Partial Information: The Drift Process as a Continuous Time Markov Chain, Finance and Stochastics, 8 (2004), 553-577.

[8] L. R. Sotomayor, and A. Cadenillas, Explicit Solutions of ConsumptionInvestment Problems in Financial Markets with Regime Switching, Mathematical Finance, 19 (2009), 251-279.

[9] R. Stockbridge, Portfolio optimization in markets having stochastic rates, In: Stochastic Theory and Control, Springer, Berlin Heidelberg (2002), 447-458.

[10] F. Venegas-Martínez, Riesgos financieros y económicos: productos derivados y decisiones económicas bajo incertidumbre, Cengage, México (2008).

[11] T. Zariphopoulou, Investment-Consumption Models with Transaction Fees and Markov-Chain Parameters, SIAM Journal on Control and Optimization, 30 (1992), 613-636.

[12] Q. Zhang and G. Yin, Nearly-optimal Asset Allocation in Hybrid Stock Investment Models, Journal of Optimization theory and Applications, 121 (2004), 419-444. 